\title{
Deleterious CHEK1 Gene Mutation
}

National Cancer Institute

\section{Source}

National Cancer Institute. Deleterious CHEK1 Gene Mutation. NCI Thesaurus. Code C134512.

A change in the nucleotide sequence of the CHEK1 gene that is associated with increased risk of disease. 